\title{
Topological D-branes and commutative algebra
}

\author{
Paul S. Aspinwall
}

\begin{abstract}
We show that questions concerning the topological B-model on a Calabi-Yau manifold in the Landau-Ginzburg phase can be rephrased in the language of commutative algebra. This yields interesting and very practical methods for analyzing the model. We demonstrate how the relevant "Ext" groups and superpotentials can be computed efficiently by computer algebra packages such as Macaulay. This picture leads us to conjecture a general description of D-branes in linear sigma models in terms of triangulated categories. Each phase of the linear sigma model is associated with a different presentation of the category of D-branes.
\end{abstract}

\section{Introduction}

As is well-known, the topological A-model is associated to the symplectic geometry of a Calabi-Yau manifold, while the B-model is associated to algebraic geometry. This, in addition to the fact that the A-model suffers from instanton corrections, makes the A-model "difficult" and the B-model "easy." But just how easy is the B-model? Suppose our Calabi-Yau manifold is a hypersurface in $\mathbb{P}^{n}$ given by a constraint $W=0$. In this case, the spectrum of closed strings and the structure of their correlation functions is given by the chiral ring [1]

$$
\frac{k\left[x_{0} \ldots x_{n-1}\right]}{\left(\partial_{0} W, \ldots, \partial_{n-1} W\right)} .
$$

For open strings we need to work harder. First we need to classify the boundary conditions, or D-branes. This is given by the (bounded) derived category of coherent sheaves on $X$ (see, for example, [2-5]). Then, given two D-branes $E$ and $F$, we may compute the Hilbert space of open strings between these D-branes from $\operatorname{Ext}^{k}(E, F)$. Certain correlation functions between these open strings form an $A_{\infty}$-structure as analyzed in [6-8]. These correlation functions are associated to the superpotential of an associated D-brane world-volume field theory. 
A principal idea in our analysis is the use of matrix factorizations to represent the D-branes. Such matrix factorizations are known to be a natural language for D-branes in the Landau-Ginzburg phase of a Calabi-Yau compactification [9-11]. The equivalence of matrix factorizations and coherent sheaves was discussed in [11-17] and then proven in [18]. The explicit form of the map between these categories was analyzed in [19]. Confronted directly, the algebra of matrix factorizations seems very unwieldy but in this paper we will show that all the necessary calculations can be reduced to questions in commutative algebra. Furthermore, commutative algebra packages such as Macaulay [20] are well suited to performing the necessary computations. Indeed, the method that Macaulay uses to compute Ext groups, as discussed in $[21]$, is uncannily well suited to our needs.

As is often the case, a better understanding of computations will give further insight into the basic structure at hand. We will see that it is natural to consider matrix factorizations of the superpotential that appears in the gauged linear $\sigma$-model rather than the superpotential of the LandauGinzburg theory. The category of D-branes will then be a quotient of this matrix factorization category. Which quotient is taken depends on which "phase" of the linear $\sigma$-model we are in. This leads to a conjectured equivalence between many different triangulated categories.

In Section 2 we will discuss topological D-branes from the point of view of matrix factorizations and, in particular, we will tackle the problem of how best to compute the Hilbert spaces of open strings between such D-branes. We believe the best way to understand this analysis is through a practical computation using Macaulay and so we give the details of a specific example.

In Section 3 we discuss the $A_{\infty}$-structure of the D-brane category. This again can be computed practically using Macaulay. We also see explicitly how to map from closed string vertex operators to open string boundarypreserving operators.

In Section 4 we see that the construction of Section 2 naturally lends itself to a general conjecture of how to construct a category of D-branes in any phase of the gauged linear $\sigma$-model. We show that this conjecture works in many cases. Finally in Section 5 we present some conclusions.

\section{Matrix factorizations}

\subsection{The D-brane category}

Let us review the construction of Kapustin and Li [9]. Consider a LandauGinzburg theory in $k^{n}$ with coordinates $x_{0}, \ldots, x_{n-1}$ and superpotential 
$W\left(x_{0}, \ldots, x_{n-1}\right)$. Physically we want the field $k$ to be equal to the complex numbers, $\mathbb{C}$. However, for computational efficiency, we also want to consider other fields.

Let $B$ denote the polynomial ring $k\left[x_{0}, \ldots, x_{n-1}\right]$. We construct the D-brane category as follows. Objects $\bar{P}$ are ordered pairs of free $B$-modules of arbitrary but equal finite rank with maps between them going in each direction:

$$
\bar{P}=\left(P_{1} \underset{p_{0}}{\stackrel{p_{1}}{\gtrless}} P_{0}\right) .
$$

The two maps satisfy the matrix factorization condition

$$
p_{0} p_{1}=p_{1} p_{0}=W . \mathbf{1} .
$$

Trivially we may also combine $p_{0}$ and $p_{1}$ to form a map

$$
p: P_{0} \oplus P_{1} \rightarrow P_{0} \oplus P_{1},
$$

where

$$
p^{2}=W .1 .
$$

A map $f: \bar{P} \rightarrow \bar{Q}$ is simply a pair of maps $f_{0}: P_{0} \rightarrow Q_{0}$ and $f_{1}: P_{1} \rightarrow$ $Q_{1}$ such that all squares commute. A map is said to be null-homotopic (or a null-homotopy) if there are maps $s_{0}: P_{0} \rightarrow Q_{1}$ and $s_{1}: P_{1} \rightarrow Q_{0}$ such that

$$
f_{0}=s_{1} p_{0}+q_{1} s_{0}, \quad f_{1}=q_{0} s_{1}+s_{0} p_{1} .
$$

The category of D-branes is given by the homotopy category obtained by identifying morphisms with maps modulo null-homotopies. The Hilbert space of open strings in the topological B-model between two branes is given by the space of morphisms in this category. Note that if either $p_{0}$ or $p_{1}$ is the identity map then the identity map $\bar{P} \rightarrow \bar{P}$ is a null-homotopy and thus $\bar{P}$ is equivalent to 0 in this category.

As is well-known, for a correspondence with Calabi-Yau manifolds, one must consider orbifolds of D-branes in a Landau-Ginzburg theory. For simplicity let us assume, for now, that the orbifold group is cyclic $G \cong \mathbb{Z}_{d}$. This group acts diagonally on the coordinates $x_{0}, \ldots, x_{n-1}$ where the eigenvalues give $B$ the structure of a graded ring. For example, the quintic threefold corresponds to a Landau-Ginzburg theory with $n=d=5$. In this case the five coordinates have equal eigenvalues and so one may declare the grade of 
each $x_{i}$ to be one. For a weighted projective space $\mathbb{P}_{\left\{w_{0}, w_{1}, w_{2}, \ldots,\right\}}^{N}$, one may use $w_{i}$ as the grade of $x_{i}$ for the corresponding graded ring $B$.

Using this grading structure, we now declare that the two free modules on $\bar{P}$ are graded B-modules. Let us define a morphism between two graded modules as a module map of degree 0 . Since $W$ has degree $d$ with respect to the grading, we cannot declare both $p_{0}$ and $p_{1}$ in (2.1) to be morphisms. Instead we may choose $p_{0}$ to be a map of degree $d$, and $p_{1}$ to be a map of degree 0 . Thus, $p_{0}$ is a morphism

$$
p_{0}: P_{0} \rightarrow P_{1}(d)
$$

where $(d)$ denotes a shift in the grading of a module, i.e., $M(d)_{m}=M_{d+m}$.

We may define the category $\operatorname{GrPair}(W)$ whose objects are pairs $\bar{P}$ of this form and whose morphisms are maps $f: \bar{P} \rightarrow \bar{Q}$ such that all squares commute.

If we then identify morphisms that differ by null-homotopies then we obtain the required D-brane category. Following [18] we denote this category $\operatorname{DGr} B(W)$.

As well as the grading, we also have the notion of a ghost number charge for each object. A shift in this charge will be denoted by square brackets [...]. As is well established (see [22] for a review) this shift gives the category of D-branes the structure of a triangulated category. It was shown in [23] that this shift functor acts on $\operatorname{DGr} B(W)$ as

$$
\bar{P}[1]=\left(P_{0}^{\stackrel{p_{0}}{\rightleftarrows} \underset{p_{1}}{\gtrless}} P_{1}(d)\right) .
$$

It follows that

$$
\bar{P}[2]=\bar{P}(d) .
$$

In this paper we will be dealing with many categories. Therefore, any mention of Hom or Ext should properly come with a subscript to denote the relevant category. We will use the convention that $\operatorname{Ext}^{j}(\mathrm{a}, \mathrm{b})=\operatorname{Hom}(\mathrm{a}, \mathrm{b}[j])$ without any subscript refers to the category of D-branes. That is, $\operatorname{Ext}^{j}(\mathrm{a}, \mathrm{b})$ is the Hilbert space of open strings from D-brane a to D-brane b shifted by ghost number $j$. 


\subsection{Construction of resolutions}

The above construction of the category $\operatorname{DGr} B(W)$ is straight-forward but, as defined, it is far from convenient to perform any computations. In this section we will review a construction of Avramov and Grayson [21] $]^{1}$ based on $[24,25]$ that turns out to be very well suited to our problem.

Again we let $B$ denote a polynomial ring $k\left[x_{0}, \ldots, x_{n-1}\right]$. For some of this paper it will suffice to assume that $B$ is singly graded. For generality, however, we will allow $B$ to be a multiply graded ring. Let $I$ be a homogeneous ideal $\left(f_{1}, f_{2}, \ldots, f_{c}\right)$ in $B$. We assume that $f_{1}, f_{2}, \ldots, f_{c}$ form a regular sequence. Now define the graded $\operatorname{ring} A=B / I$.

If $B$ is singly and equally graded, then the condition of a regular sequence is equivalent to the statement that the projective variety defined by $f_{1}=$ $f_{2}=\ldots=f_{c}=0$ in $\mathbb{P}^{n-1}$ is a complete intersection. Let $X=\operatorname{Proj} A$ denote this complete intersection. More generally we would define $X$ as a complete intersection in a toric variety.

Consider a finitely generated graded $A$-module $M$. Equivalently $M$ may be regarded as a $B$-module which is annihilated by $I$. We wish to study the question of how to compute a free resolution of the $A$-module $M$ :

$$
\cdots \longrightarrow F_{2} \longrightarrow F_{1} \longrightarrow F_{0} \longrightarrow M \longrightarrow 0
$$

where $F_{j}$ is a free module $A\left(a_{j, 0}\right) \oplus A\left(a_{j, 1}\right) \oplus \ldots$ and $a_{j, s}$ are integers denoting "twists" of $A .^{2}$ This will allow computations of $\operatorname{Ext}_{A}$ groups.

Spec $A$ is the affine variety defined by $f_{1}=f_{2}=\cdots=f_{c}=0$ in $\mathbb{C}^{n}$. If $\operatorname{Spec} A$ is smooth then we are guaranteed to have a finite free resolution. It turns out that the modules of interest to us are the ones with no finite free resolution. We are interested in the case where $\operatorname{Spec} A$ has an isolated singularity at the origin, and hence $X$ is smooth.

Since $\mathbb{C}^{n}$ is trivially smooth, as a $B$-module $M$ will have a finite free resolution

$$
\stackrel{d_{C}}{\longrightarrow} C_{2} \stackrel{d_{C}}{\longrightarrow} C_{1} \stackrel{d_{C}}{\longrightarrow} C_{0} \stackrel{r}{\longrightarrow} M \longrightarrow 0
$$

where each $C_{i}$ is a finitely generated free $B$-module.

\footnotetext{
${ }^{1}$ In order to retain the same notation as [18] we have interchanged the roles of $A$ and $B$ in this reference.

${ }^{2}$ This notation is for a singly graded ring. The multiple grading case will require multiple twists for each summand.
} 
Define the finitely generated free $B$-module

$$
C=\bigoplus_{i} C_{i} .
$$

Suppose $A$ is multiply graded by $r$ integers. Then $C$ has $r+1$ gradings. The extra grading is given by the subscripts in (2.11). We will refer to this latter grading as the homological grading. The other $r$ gradings will be called the ring gradings. The map $d_{C}$ in (2.10) then has homological degree -1 and all the ring degrees are 0 .

Recall that $c$ denotes the number of equations defining our complete intersection. Introduce $c$ variables $X_{1}, \ldots, X_{c}$ each with a homological degree of -2 and ring degrees of $-\operatorname{deg} f_{i}$. Now define the multiply graded ring

$$
S=B\left[X_{1}, X_{2}, \ldots, X_{c}\right] .
$$

Set $D=\operatorname{Hom}_{B}(S, B)$, the dual of $S . D$ is naturally endowed with the structure of an algebra known as the divided powers algebra. We refer to Appendix A2 of [26] for more details. We will not need any particular knowledge of this divided powers structure here.

Let $\gamma$ be a multi-index $\left(\gamma_{1}, \ldots, \gamma_{c}\right)$ in $^{3} \mathbb{N}^{c}$ and denote the monomial $X_{1}^{\gamma_{1}} X_{2}^{\gamma_{2}} \ldots$ by $X^{\gamma}$. Let $|\gamma|=\sum_{i} \gamma_{i}$. Denote the origin $(0,0, \ldots)$ by $o$ and a basis vector $(0,0, \ldots, 1, \ldots, 0)$ by $\epsilon_{i}$. We copy the following theorem from [21]:

Theorem 2.1. There exists a set of homogeneous B-linear maps

$$
d_{\gamma}: C \rightarrow C,
$$

for all $\gamma \in \mathbb{N}^{c}$ with homological degree $2|\gamma|-1$ and ring degree 0 , such that

$$
\begin{aligned}
d_{o} & =d_{C}, \\
\left\{d_{o}, d_{\gamma}\right\} & = \begin{cases}-f_{i} . \mathbf{1}_{C}, & \text { if } \gamma=\epsilon_{i}, i=1, \ldots, c, \\
-\sum_{\substack{\alpha+\beta=\gamma \\
\alpha, \beta \neq o}} d_{\alpha} d_{\beta}, & \text { otherwise. }\end{cases}
\end{aligned}
$$

These induce a B-linear map of homological degree -1 and ring degree 0

$$
d_{C D}: C \otimes_{B} D \rightarrow C \otimes_{B} D,
$$

\footnotetext{
${ }^{3} \mathbb{N}$ contains 0.
} 
given by

$$
\left.d_{C D}(x \otimes y)=\sum_{\gamma \in \mathbb{N}^{c}} d_{\gamma}(x) \otimes\left(X^{\gamma}\right\lrcorner y\right) .
$$

The symbol $\lrcorner$ denotes the obvious contraction map between $S$ and $D$. We refer to [21] for a more detailed explanation.

One can then show the following:

Theorem 2.2. The map $d_{C D}: C \otimes_{B} D \rightarrow C \otimes_{B} D$ defined above satisfies

$$
d_{C D}^{2}=-f .1_{C \otimes D},
$$

where

$$
f=\sum_{i=1}^{c} f_{i} X_{i}
$$

This is also proven in [21].

Most importantly we have the following, again copied from [21]:

Theorem 2.3. Set $D^{\prime}=D \otimes_{B} A$ and $y^{\prime}=y \otimes 1$ for $y \in D$. The map

$$
\partial: C \otimes_{B} D^{\prime} \rightarrow C \otimes_{B} D^{\prime}
$$

given by

$$
\left.\partial\left(x \otimes y^{\prime}\right)=\sum_{\gamma \in \mathbb{N}^{c}} d_{\gamma}(x) \otimes\left(X^{\gamma}\right\lrcorner y\right)^{\prime}
$$

is an A-linear map of homological degree -1 . This, together with the map

$$
q^{\prime}: C \otimes_{B} D^{\prime} \rightarrow M
$$

given by

$$
q^{\prime}\left(x \otimes y^{\prime}\right)= \begin{cases}y \cdot r(x), & \text { if } \operatorname{deg}\left(y^{\prime}\right)=0 \\ 0, & \text { otherwise }\end{cases}
$$

is a resolution of $M$ by free $A$-modules.

Note that the map $r$ refers to the one in $(2.10)$ and $\operatorname{deg}\left(y^{\prime}\right)$ refers to the homological degree. 
This yields a very practical construction of the free resolution of $M$ that we will need. In particular, this algorithm can be efficiently implemented in Macaulay 2 as described in [21].

Note that such a free resolution is always infinitely long since $D$ has unbounded homological degree. However, $\partial$ is represented by a finitedimensional matrix with entries in the ring $S$.

Given such a resolution of $M$, it is easy to compute the groups $\operatorname{Ext}_{A}(M, N)$ for any $A$-module $N$. Note that the action of $S$ on the free resolution descends to $\operatorname{Ext}_{A}(M, N)$ and thus $\operatorname{Ext}_{A}(M, N)$ is an $S$-module. Again $\bigoplus_{k} \operatorname{Ext}_{A}^{k}(M, N)$ can typically be infinite-dimensional but it can always be presented as a finitely generated $S$-module. The variables $X_{i}$ yield maps

$$
X_{i}: \operatorname{Ext}_{A}^{j}(M, N) \rightarrow \operatorname{Ext}_{A}^{j+2}(M, N) .
$$

As such, the homological degree we have defined is negative to what one might normally use in the derived category. This is not surprising since we graded (2.10) according to homology rather than cohomology.

Unfortunately it is not these Ext groups that compute the Hilbert spaces of open strings. Having said that, the construction is extremely relevant, as we now describe.

\subsection{A quotient category}

For the time being, let us restrict to the case of a hypersurface, $c=1$. It is tempting to note the similarity between (2.4) and (2.17). The only difference is the appearance of the extra factor $-X_{1}$ in $(2.17)$. We will now show that we may convert the construction of Section 2.2 to that required in Section 2.1 simply by setting $X_{1}$ equal to -1 .

Let us also restrict to the case where $A$ is singly graded and $f_{1}=W$ has degree $d$. The homological and ring grading of $X_{1}$ is then equal to $(-2,-d)$. The only way we could set $X_{1}$ equal to -1 is if we identify the homological and ring grading accordingly. Indeed we would require $M[2]=M(d)$ exactly like in (2.8). This is further evidence that we are on the right track.

In order to prove our assertion we need to introduce various categories and consider functors between them.

Let $\mathrm{Ch}(\mathrm{gr}-A)$ be the category whose objects are bounded chain complexes of graded $A$-modules and whose morphisms are chain maps of degree 0 . Recall that $\operatorname{GrPair}(W)$ is the category of matrix factorizations (without modding out by homotopy) defined in Section 2.1. We attempt to define a functor from $\mathrm{Ch}(\mathrm{gr}-A)$ to $\operatorname{GrPair}(W)$ as follows: 
- Any object $M^{\bullet}$ in $\mathrm{Ch}(\mathrm{gr}-A)$ can be represented by a bounded complex of $B$-modules annihilated by $W$.

- This complex may then be represented by a finite free resolution, i.e., a finite complex $C^{\bullet}$ of free $B$-modules. In other words, we have a chain map $C^{\bullet} \rightarrow M^{\bullet}$ which is a quasi-isomorphism.

- Now take this chain complex $C^{\bullet}$ and form the direct sum of its elements to form $C$ as in (2.11) as well as the differential $d_{C}: C \rightarrow C$.

- Then apply the construction of Theorem 2.1 to form a map $p: C \rightarrow C$ where

$$
p(x)=\sum_{\gamma \in \mathbb{N}}(-1)^{\gamma} d_{\gamma}(x) .
$$

This is essentially the same as (2.16) where $D$ has been collapsed to $B$ (with an alternating sign) and thus we have effectively set $X_{1}=-1$. The relation (2.17) becomes $p^{2}=W$.

- Decomposing $C$ by homological degree we put $P_{0}=C^{\text {even }}$ and $P_{1}=$ $C^{\text {odd }}$, and $p$ decomposes into two maps $p_{0}$ and $p_{1}$ between these modules. Thus we obtain an object in $\operatorname{GrPair}(W)$.

The only problem with the above construction is that we required a choice of free resolution $C^{\bullet}$. Naturally the solution to this problem is to go to the homotopy category. It is easy to show that a chain homotopy in $\operatorname{Ch}(\operatorname{gr}-A)$ will be mapped to a homotopy in $\operatorname{GrPair}(W)$ by the above construction. Let $K(\operatorname{gr}-A)$ denote the category whose objects are complexes of graded free $A$-modules and whose morphisms are chain maps modulo chain homotopies. We also impose the following finiteness condition of $K(\mathrm{gr}-A)$. The complexes are bounded to the right but may be infinite to the left. However, they can be finitely represented by $S$-modules as in Section 2.2 .

This yields a well-defined functor

$$
K(\operatorname{gr}-A) \rightarrow \operatorname{DGr} B(W)
$$

We now claim that the category $K(\operatorname{gr}-A)$ is equivalent to $\mathbf{D}(\operatorname{gr}-A)$, the bounded derived category of $A$-modules. This may be proven by using, for example, the result of Section 3.10 of [27] combined with the fact that any bounded complex of $A$-modules has a free resolution satisfying the above finiteness condition on $K(\operatorname{gr}-A)$. 
This yields a functor

$$
G^{\prime}: \mathbf{D}(\operatorname{gr}-A) \rightarrow \operatorname{DGr} B(W)
$$

One may check that this is an exact functor between triangulated categories.

For the next step we need to recall the definition of a quotient triangulated category (see [28] for example). Given a triangulated category D and full triangulated subcategory $\mathrm{N}$, we define the quotient $\mathrm{D} / \mathrm{N}$ as follows. The objects in $\mathrm{D} / \mathrm{N}$ are the same as the objects in $\mathrm{D}$. Consider the set of morphisms $\Sigma$ in $\mathrm{D}$ whose mapping cones lie in $\mathrm{N}$. In other words $f: \mathrm{a} \rightarrow \mathrm{b}$ lies in $\Sigma$ if and only if we have a distinguished triangle

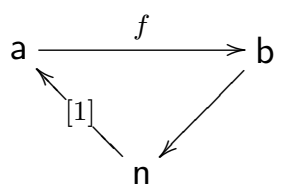

where $\mathrm{n}$ is an object in $\mathrm{N}$. The octahedral axiom can be used to show that $\Sigma$ is multiplicatively closed. The morphisms in $\mathrm{D} / \mathrm{N}$ are then defined by "localizing" on the set $\Sigma$. That is, we invert the elements of $\Sigma$ in the same way that quasi-isomorphisms are inverted in defining the derived category.

Note, in particular, that the zero map $0 \rightarrow \mathrm{n}$ is in $\Sigma$ so that any element of $\mathrm{N}$ is isomorphic to zero in $\mathrm{D} / \mathrm{N}$.

Let $\mathfrak{P e r} \mathfrak{f}(A)$ be the full subcategory of $\mathbf{D}(\operatorname{gr}-A)$ consisting of objects that may be represented by finite-length complexes of free $A$-modules of finite rank. The category $\mathbf{D}_{\mathrm{Sg}}^{\mathrm{gr}}(A)$ is then defined as the quotient (in the above sense)

$$
\mathbf{D}_{\mathrm{Sg}}^{\mathrm{gr}}(A)=\frac{\mathbf{D}(\operatorname{gr}-A)}{\mathfrak{P e r} \mathfrak{f}(A)}
$$

$\mathbf{D}_{\mathrm{Sg}}^{\mathrm{gr}}(A)$ has the following universal property. If $U: \mathbf{D}(\operatorname{gr}-A) \rightarrow \mathcal{C}$ is an exact functor, for some triangulated category $\mathcal{C}$, such that $U(M) \cong 0$ for any object $M$ in $\mathfrak{P e r f}(A)$, then $U$ factors through $\mathbf{D}_{\mathrm{Sg}}^{\mathrm{gr}}(A)$.

The functor $G^{\prime}$ in (2.26) indeed has the above property. Let us consider applying $G^{\prime}$ to the $A$-module $A$ itself. First we represent $A$ by a free resolution of $B$-modules. This is the complex

$$
B(-d) \stackrel{W}{\rightarrow} B
$$


Thus $C=B(-d) \oplus B$. It is then easy to show that $d_{1}$ maps $B$ to $B(-d)$ as a multiplication by -1 and $d_{j}=0$ for $j>1$. Thus

$$
p=d_{0}-d_{1}=\left(\begin{array}{cc}
0 & 1 \\
W & 0
\end{array}\right) .
$$

That is, $A$ is mapped to the trivial matrix factorization $W=W .1$. The identity map of this matrix factorization to itself is a null-homotopy. Thus, this object is isomorphic to zero in $\operatorname{DGr} B(W)$. That is, $G^{\prime}(A) \cong 0$. Since $G^{\prime}$ is an exact functor, it follows immediately that $G^{\prime}$ applied to any finite complex of finitely generated free $A$-modules yields zero. We have therefore defined an induced functor

$$
G: \mathbf{D}_{\mathrm{Sg}}^{\mathrm{gr}}(A) \rightarrow \operatorname{DGr} B(W) .
$$

This functor is actually an equivalence of categories. To see this one can construct an inverse functor as follows. Consider the functor from $\operatorname{GrPair}(W)$ to the category of graded $A$-modules given by the map Coker $\left(p_{1}\right)$. As shown in [18] this induces a functor

$$
F: \operatorname{DGr} B(W) \rightarrow \mathbf{D}_{\mathrm{Sg}}^{\mathrm{gr}}(A) .
$$

Now consider an object $\bar{P}$ in DGr $B$. Applying $G F$ to $\bar{P}$ will produce another matrix factorization which is typically larger than $\bar{P}$. However, both $\bar{P}$ and $G F(\bar{P})$ represent free resolutions of the same module and so are equivalent in the homotopy category $\operatorname{DGr} B(W)$. Thus $G F \cong \mathbf{1}$. Similarly applying $F G$ to a complex of modules yields a complex of modules that differs from the original by a finite complex of free modules and hence $F G \cong 1$. Essentially, by Eisenbud's construction [24] the original complex has a free resolution which will eventually become 2-periodic to the left, whereas $F G$ applied to this complex has a free resolution that is immediately the same 2-periodic resolution.

We have therefore proven the following:

Theorem 2.4. The functor

$$
G: \mathbf{D}_{\mathrm{Sg}}^{\mathrm{gr}}(A) \cong \operatorname{DGr} B(W)
$$

is an equivalence of triangulated categories. 
That these two categories are equivalent is not a new result - it was proven in [18]. What is useful to us is that the equivalence is induced by the explicit functor $G$.

What we have shown is that the quotient $\mathbf{D}(\operatorname{gr}-A) \rightarrow \mathbf{D}_{\mathrm{Sg}}^{\mathrm{gr}}(A)$ is essentially performed by setting $X_{1}=-1$. To be more precise, let $M^{\bullet}$ and $N^{\bullet}$ be two objects in $\mathbf{D}(\operatorname{gr}-A)$. As shown in [21] and Section 2.2, the group $\bigoplus_{j, n} \operatorname{Hom}_{\mathbf{D}(\operatorname{gr}-A)}\left(M^{\bullet}, N^{\bullet}[j](n)\right)$ is a finitely generated $S$-module. Let $B$ be regarded as an $S$-module where the action of $X_{1}$ is multiplication by -1 . Theorem 2.4 shows that

$$
\bigoplus_{j, n} \operatorname{Hom}_{\mathbf{D}_{\mathrm{Sg}}^{\mathrm{gr}}(A)}\left(M^{\bullet}, N^{\bullet}[j](n)\right) \cong \bigoplus_{j, n} \operatorname{Hom}_{\mathbf{D}(\mathrm{gr}-A)}\left(M^{\bullet}, N^{\bullet}[j](n)\right) \otimes_{S} B .
$$

\subsection{A 2-brane on a twisted cubic}

To clarify the construction of the previous section and to show how to practically implement the procedure we give an example using Macaulay 2.

We will give an example that is fairly simple so that we do not need to do any extra programming. Having said that, it is sufficiently complicated that computations "by hand" would be fairly awkward.

Let $X$ be the quintic three fold defined by the equation

$$
W=x_{0}^{3} x_{1} x_{2}+x_{1}^{3} x_{2}^{2}+x_{1}^{2} x_{2}^{3}-x_{0} x_{2}^{4}-x_{0}^{4} x_{3}-x_{1}^{4} x_{3}+x_{3}^{4} x_{4}+x_{4}^{5}
$$

We would like to consider D-branes wrapping the "twisted" cubic rational curve defined by the ideal

$$
I=\left(x_{1}^{2}-x_{0} x_{2}, x_{2}^{2}-x_{1} x_{3}, x_{1} x_{2}-x_{0} x_{3}, x_{4}\right)
$$

We will compute the dimensions of the Hilbert spaces of open strings beginning and ending on this D-brane, together with certain "twists" of the Dbrane.

Let $B=k\left[x_{0}, \ldots, x_{4}\right]$ and let $A=B /(W)$ as above. Then $X=\operatorname{Proj} A$. The sheaf supported on the cubic curve is then associated to the $A$-module $M=A / I$.

Usually, given a sheaf (or complex of sheaves) in the geometric picture of D-branes, one needs to go through a nontrivial process to obtain an $A$-module in $\mathbf{D}_{\mathrm{Sg}}^{\mathrm{gr}}(A)$ that represents the same $\mathrm{D}$-brane in the LandauGinzburg picture. The precise algorithm was given in [19]. Having said that, it was shown in [19] that this process is trivial for projectively normal 
rational curves. Thus the $A$-module $M$ correctly represents our 2-brane in $\mathbf{D}_{\mathrm{Sg}}^{\mathrm{gr}}(A)$.

Let us proceed with our example in Macaulay 2. For the first few lines of input we will suppress printing the output. What follows is the basic setup defining the rings and modules above. Note that, as usual in Macaulay, we let $k$ be a finite field rather than the complex numbers to improve the efficiency of the computation. As long as there are no unfortunate coincidences between the coefficients in the polynomials that Macaulay manipulates and the characteristic of $k$, this should not affect the results.

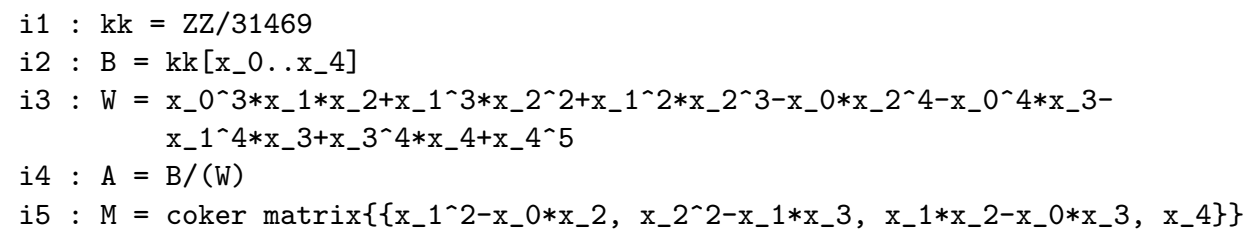

Now we use the internal Macaulay routine described in [21] to compute the $S$-module $\operatorname{Ext}_{A}^{*}(M, M)$ :

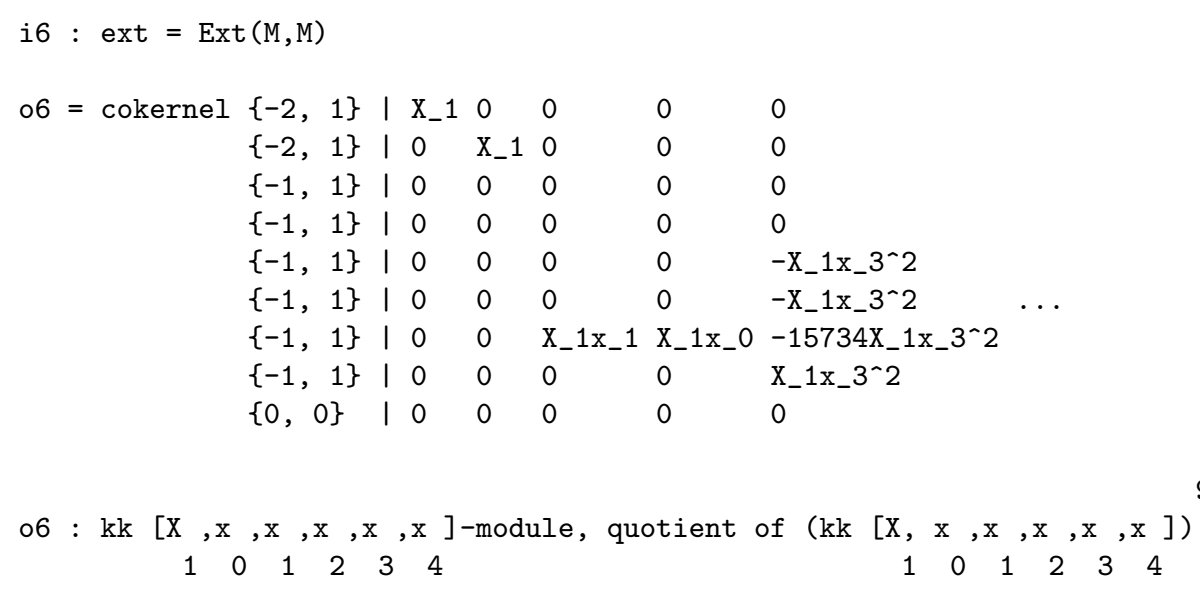

The output above is quite large and we have suppressed most of it. The first column of the output represents the bi-degrees of the generators of this module. The first degree is the homological degree discussed in the previous section and the second degree is the original degree associated to our graded ring $B$. For example, the generator associated to the first row is an element of $\operatorname{Ext}_{A}^{2}(M, M(1))$. The final row represents the identity map. The 44 columns of the matrix in the rest of the output represent relations between these generators. 
Next we need to pass to the quotient category $\mathbf{D}_{\mathrm{Sg}}^{\mathrm{gr}}(A)$ by setting $X_{1}=-1$. When we do this, we will collapse the bigrading to a single grading satisfying $M[2]=M(5)$. To keep track of this single grading we map an old grade of $(a, b)$ to the single grade $-5 a+2 b$. Thus the degrees of $x_{0}, \ldots, x_{4}$ in our new ring will be 2 . We denote this new ring B2.

The following code sets pr equal to the map whose cokernel defines $\operatorname{Ext}_{A}^{*}(M, M)$ above and we define our rings $S$ and B2.

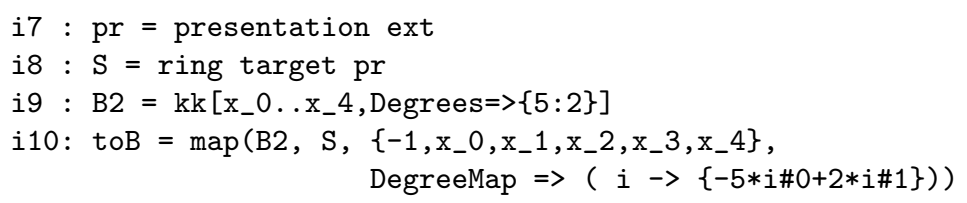

The last line above is the heart of our algorithm. It defines a ring map that sets $X_{1}=-1$ and defines how we map degrees. It is now simple to compute the Ext's in the quotient category $\mathbf{D}_{\mathrm{Sg}}^{\mathrm{gr}}(A)$ by constructing the tensor product as in (2.34):

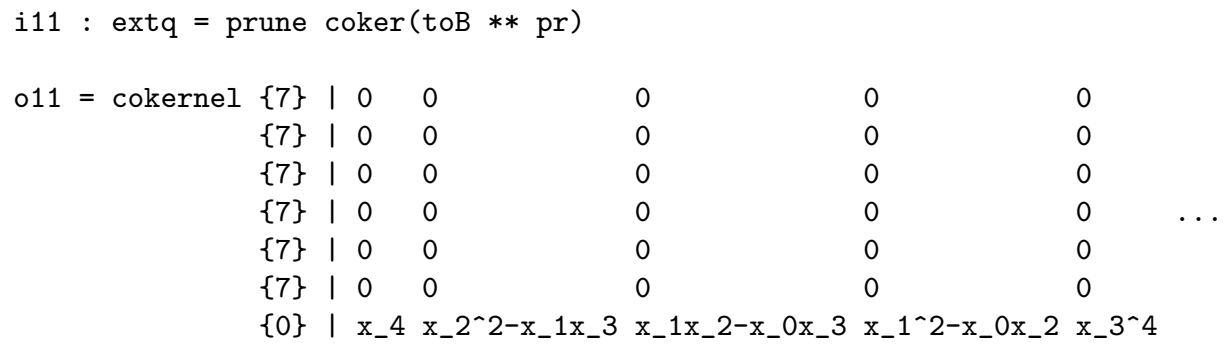

o11 : B2-module, quotient of B2

Finally we may compute the dimensions of Hilbert spaces of open strings by computing the dimensions of the above module at specific degrees. This, of course, is the Hilbert function.

i12 : $\operatorname{apply}(20, i \rightarrow \operatorname{hilbertFunction}(i, \operatorname{extq}))$

$012=\{1,0,4,0,7,0,10,6,6,10,0,7,0,4,0,1,0,0,0,0\}$

012 : List

The above list represents the dimensions of $\left.\operatorname{Hom}_{\mathbf{D}_{\mathrm{Sg}}^{\mathrm{gr}}(A)}(M, M\langle i\rangle)\right)$ for $i=0 \ldots 19$, where we use angle brackets to represent twisting with respect to the single grading we have defined. That is,

$$
\operatorname{Ext}^{k}(M, M(r))=\operatorname{Hom}_{\mathbf{D}_{\mathrm{Sg}}^{\mathrm{gr}}(A)}(M, M\langle 5 k+2 r\rangle) .
$$


Note that Serre duality implies $\left.\operatorname{Hom}_{\mathbf{D}_{\mathrm{Sg}}^{\mathrm{gr}}(A)}(M, M\langle i\rangle)\right) \cong \operatorname{Hom}_{\mathbf{D}_{\mathrm{Sg}}^{\mathrm{gr}}(A)}(M, M$ $\langle 15-i\rangle)$ ), which is consistent with the above output. For open strings beginning and ending on the same untwisted 2-brane $M$ we immediately see

$$
\begin{aligned}
& \operatorname{Ext}^{0}(M, M)=\mathbb{C}, \quad \operatorname{Ext}^{1}(M, M)=0, \\
& \operatorname{Ext}^{2}(M, M)=0, \quad \operatorname{Ext}^{3}(M, M)=\mathbb{C} .
\end{aligned}
$$

This shows that our twisted cubic curve has normal bundle $\mathscr{O}(-1) \oplus \mathscr{O}(-1)$.

We could also compute open string Hilbert spaces between $M$ and twists of $M$. (Physically this twisting corresponds to monodromy around the Landau-Ginzburg limit as discussed in [19].) For example, $\operatorname{Ext}^{1}(M$, $M(1))=\mathbb{C}^{6}$.

\section{Superpotentials}

The derived category associated to topological D-branes is endowed with an $A_{\infty}$-structure as discussed in $[7,8,13,29-31]$. This $A_{\infty}$-structure also manifests itself as an effective superpotential in the D-brane world-volume theory. Viewing D-branes in the geometric phase as complexes of coherent sheaves, this $A_{\infty}$-structure can be quite tricky to compute. It is associated with obstructions to deformations of the associated geometric objects.

Now that we have a much more computationally favorable setting in the Landau-Ginzburg phase, we should have an easier time discovering the $A_{\infty}$-structure and thus computing superpotentials. Let us review how we perform this computation.

Let $P$ denote $P_{0} \oplus P_{1}$. Our D-brane is a map $p: P \rightarrow P$ satisfying $p^{2}=$ $W$. Now consider another map $a: P \rightarrow P$ which has a definite parity, i.e., the map acts within $P_{0}$ and $P_{1}$ or it exchanges them. (The literature such as [10] often calls even parity maps "bosonic" and odd parity maps "fermionic".) We denote the parity $(-1)^{a}$. Now define a differential

$$
d a=a p-(-1)^{a} p a .
$$

It follows that $d^{2}=0$ and the cohomology of this operator is exactly the open string Hilbert spaces we discussed in Section 2.1.

We also have a simple composition of maps. That is, if $a$ and $b$ are both maps $P \rightarrow P$ then so is $a b$. This composition satisfies the Leibniz rule with respect to the differential. Thus we have the structure of a $\mathbb{Z}_{2}$-graded differential graded algebra. For the Landau-Ginzburg orbifold we consider graded modules as in Section 2.1. As before, this can be used to extend the 
$\mathbb{Z}_{2}$-grading to a $\mathbb{Z}$-grading. (In the language of homological algebra we are defining the "Yoneda product" between the corresponding Ext groups.)

As explained in [8,32], for example, a differential graded algebra gives rise to an $A_{\infty}$-structure on the cohomology of this algebra, which is unique up to an $A_{\infty}$-isomorphism thanks to Kadeishvili's theorem [33] as we now review.

An $A_{\infty}$-algebra $\mathscr{A}$ comes equipped with a set of products

$$
m_{k}: \mathscr{A}^{\otimes k} \rightarrow \mathscr{A}
$$

satisfying particular relations (see [32] for example). An $A_{\infty}$-morphism from $\mathscr{A}$ to $\mathscr{B}$ is a collection of maps $f_{k}: \mathscr{A}^{\otimes k} \rightarrow \mathscr{B}$ satisfying

$\sum_{r+s+t=n}(-1)^{r+s t} f_{u}\left(\mathbf{1}^{\otimes r} \otimes m_{s} \otimes \mathbf{1}^{\otimes t}\right)=\sum_{\substack{1 \leq r \leq n \\ i_{1}+\cdots+i_{r}=n}}(-1)^{q} m_{r}\left(f_{i_{1}} \otimes f_{i_{2}} \otimes \cdots \otimes f_{i_{r}}\right)$,

for any $n>0$ and $u=n+1-s$. The sign on the right is given by

$$
q=(r-1)\left(i_{1}-1\right)+(r-2)\left(i_{2}-1\right)+\ldots+\left(i_{r-1}-1\right) .
$$

A differential graded algebra $\mathscr{B}$ is trivially an $A_{\infty}$-algebra where $m_{1}$ is the differential, $m_{2}$ is the product and all higher $m_{k}$ 's vanish. Let $H(\mathscr{B})$ denote the cohomology of $\mathscr{B}$ and choose an embedding $i: H(\mathscr{B}) \rightarrow \mathscr{B}$ which we set equal to $f_{1}$. One may then iteratively apply (3.3) for increasing values of $n$ to define higher $f_{k}$ 's and $m_{k}$ 's on $H(\mathscr{B})$ to define an $A_{\infty}$-structure on $H(\mathscr{B})$. As stated above, this can be shown to be unique up to an $A_{\infty}$-isomorphism.

Given a topological field theory of open strings we may use the BRST operator $Q$ as the differential and the joining of open strings as the product. The resulting $A_{\infty}$-structure on the Hilbert spaces of open strings then yields the desired one associated to the superpotential, at least up to an $A_{\infty}$-isomorphism. This ambiguity seems to be unavoidable without using information beyond the topological field theory.

Anyway, we are clearly in a position to compute the $A_{\infty}$-structure. We simply need a representative map $P \rightarrow P$ for each basis element of $\operatorname{Ext}^{k}(P, P)$. Unfortuantely, a naïve approach to the problem can easily come up against computational limits.

Suppose we consider a D-brane such that $C$, as constructed in Section 2.2 is of rank $N$. Thus $p$ is an $N \times N$ matrix. Now consider the space of all homomorphisms $C \rightarrow C$. Such homomorphisms are also $N \times N$ matrices and thus we have an $N^{2}$-dimensional space of Hom's. It follows that the differential $d$ in (3.1) is represented by an $N^{2} \times N^{2}$ matrix. Since $N$ can 
easily be of the order of 100 for relatively simple D-branes, we see that this direct method can be very unwieldy.

This is, of course, not how Macaulay computed the Ext groups in the last example. In order to compute $\operatorname{Ext}_{A}(M, M)$, we need to find a resolution $C$ of $M$ and then compute the cohomology of $\operatorname{Hom}(C, M)$ which is just an $N \times N$ matrix. Thus, Macaulay would represent an element of $\operatorname{Ext}_{A}(M, M)$ by an element of $\operatorname{Hom}(C, M)$. Because of the properties of projective modules, we may lift $^{4}$ to the desired map from $C$ to $C$ :

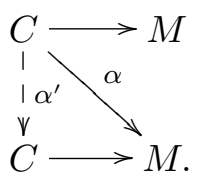

If we are considering an object in the derived category that cannot be represented by a single $A$-module $M$, then we may apply the mapping cone construction to build the necessary map.

Let us illustrate the procedure for a very simple example. Actually this example has already been analyzed in [34] where the computational complexity was reduced by using tensor product structures rather than the approach we use here. Our method can be applied to any D-brane but we will stick to this easy case for the reader's benefit. Here we need to do a little more Macaulay programing as we need to get "inside" the code for Ext listed in [21] to find the map $p$. We spare the reader the details.

Let $B=k\left[x_{0}, \ldots, x_{4}\right], A=B /(W)$ and $X=\operatorname{Proj} A$ be the Fermat quintic given by

$$
W=x_{0}^{5}+x_{1}^{5}+x_{2}^{5}+x_{3}^{5}+x_{4}^{5}
$$

Again we will consider a rational curve to avoid the complexities of mapping between $\mathbf{D}(X)$ and $\mathbf{D}_{\mathrm{Sg}}^{\mathrm{gr}}(A)$. The one we choose is given by $x_{0}+x_{1}=x_{2}+$ $x_{3}=x_{4}=0$. That is, our 2-brane is given by the $A$-module

$$
M=\frac{A}{\left(x_{0}+x_{1}, x_{2}+x_{3}, x_{4}\right)} .
$$

\footnotetext{
${ }^{4}$ There is a subtlety here. In order to lift this map systematically we require $d_{C}$ to have a strictly negative homological degree. This is true in the category $\mathbf{D}(\operatorname{gr}-A)$ but is no longer true when we set $X_{1}=-1$. Thus we find representatives of our open strings as matrices before we set $X_{1}=-1$.
} 
The map $p$ is given by the following matrix:

$$
p=\left(\begin{array}{cccccccc}
0 & a_{0} & a_{1} & a_{2} & 0 & 0 & 0 & 0 \\
b_{0} & 0 & 0 & 0 & -a_{1} & -a_{2} & 0 & 0 \\
b_{1} & 0 & 0 & 0 & a_{0} & 0 & -a_{2} & 0 \\
b_{2} & 0 & 0 & 0 & 0 & a_{0} & a_{1} & 0 \\
0 & -b_{1} & b_{0} & 0 & 0 & 0 & 0 & a_{2} \\
0 & -b_{2} & 0 & b_{0} & 0 & 0 & 0 & -a_{1} \\
0 & 0 & -b_{2} & b_{1} & 0 & 0 & 0 & a_{0} \\
0 & 0 & 0 & 0 & b_{2} & -b_{1} & b_{0} & 0
\end{array}\right),
$$

where $a_{0}=x_{0}+x_{1}, a_{1}=x_{2}+x_{3}, a_{2}=x_{4}, b_{0}=x_{0}^{4}-x_{0}^{3} x_{1}+x_{0}^{2} x_{1}^{2}-x_{0} x_{1}^{3}+$ $x_{1}^{4}, b_{1}=x_{2}^{4}-x_{2}^{3} x_{3}+x_{2}^{2} x_{3}^{2}-x_{2} x_{3}^{3}+x_{3}^{4}$ and $b_{2}=x_{4}^{4}$.

Copying the procedure in Section 2.4 we obtain a particularly easy answer for $\operatorname{Ext}(M, M)$. There are only two generators. One is given by the identity map $C \rightarrow C$ so we will call it $\mathbf{1}$. The other generator has grade 3 and we call it $g . g$ is easily determined to be

$$
g=\left(\begin{array}{cccccccc}
0 & 0 & 0 & 1 & 0 & 0 & 0 & 0 \\
0 & 0 & 0 & 0 & 0 & -1 & 0 & 0 \\
0 & 0 & 0 & 0 & 0 & 0 & -1 & 0 \\
-x_{4}^{3} & 0 & 0 & 0 & 0 & 0 & 0 & 0 \\
0 & 0 & 0 & 0 & 0 & 0 & 0 & 1 \\
0 & x_{4}^{3} & 0 & 0 & 0 & 0 & 0 & 0 \\
0 & 0 & x_{4}^{3} & 0 & 0 & 0 & 0 & 0 \\
0 & 0 & 0 & 0 & -x_{4}^{3} & 0 & 0 & 0
\end{array}\right)
$$

Macaulay tells us that, for the $B$-module $\operatorname{Ext}(M, M)$ both generators 1 and $g$ are independently annihilated by the ideal

$$
I=\left(x_{0}+x_{1}, x_{2}+x_{3}, x_{4}, x_{0}^{4}, x_{2}^{4}\right) .
$$

This immediately gives us the following:

$$
\begin{aligned}
& \operatorname{Ext}^{0}(M, M)=\mathbb{C} \quad \text { generated by } \mathbf{1}, \\
& \operatorname{Ext}^{1}(M, M)=\mathbb{C}^{2} \quad \text { generated by } x_{0} g \text { and } x_{2} g, \\
& \operatorname{Ext}^{2}(M, M)=\mathbb{C}^{2} \quad \text { generated by } x_{0}^{2} x_{2}^{3} \mathbf{1} \text { and } x_{0}^{3} x_{2}^{2} \mathbf{1}, \\
& \operatorname{Ext}^{3}(M, M)=\mathbb{C} \quad \text { generated by } x_{0}^{3} x_{2}^{3} g .
\end{aligned}
$$

We can now build the $A_{\infty}$-structure. It is easy to prove that $\mathbf{1}$ acts as the identity for $m_{2}$ and that $m_{k}$ vanishes for $k>2$ if any of the entries are 
equal to 1 . So the only $A_{\infty}$-products we need to compute are $m_{k}(g, g, \ldots, g)$ for all $k$.

Putting $n=2$ in (3.3) gives

$$
i m_{2}(g, g)=d f_{2}(g, g)+g \cdot g
$$

But $g^{2}=-x_{4}^{3} \mathbf{1}$. Since $\mathbf{1}$ is killed by $x_{4}$ in $\operatorname{Ext}(M, M)$, we see that $m_{2}(g, g)=0$. Saying that $\mathbf{1}$ is killed by $x_{4}$ means that $x_{4} \mathbf{1}$ is a null-homotopic map. It is again straight-forward using the same techniques we have already employed to compute this null-homotopy. We find $x_{4} \mathbf{1}=q p+p q$, where

$$
q=\left(\begin{array}{cccccccc}
0 & 0 & 0 & 0 & 0 & 0 & 0 & 0 \\
0 & 0 & 0 & 0 & 0 & 0 & 0 & 0 \\
0 & 0 & 0 & 0 & 0 & 0 & 0 & 0 \\
1 & 0 & 0 & 0 & 0 & 0 & 0 & 0 \\
0 & 0 & 0 & 0 & 0 & 0 & 0 & 0 \\
0 & -1 & 0 & 0 & 0 & 0 & 0 & 0 \\
0 & 0 & -1 & 0 & 0 & 0 & 0 & 0 \\
0 & 0 & 0 & 0 & 1 & 0 & 0 & 0
\end{array}\right)
$$

Thus $f_{2}(g, g)=x_{4}^{2} q$. At the next step $n=3$ yields $^{5}$

$$
i m_{3}(g, g, g)=-g \cdot f_{2}(g, g)-f_{2}(g, g) \cdot g+d f_{3}(g, g, g)
$$

But $q g+g q=\mathbf{1}$, which implies $m_{3}(g, g, g)=0$ and $f_{3}(g, g, g)=x_{4} q$. Similarly $m_{4}(g, g, g, g)=0$ and $f_{4}(g, g, g, g)=-q$. For $n=5$ we obtain

$i m_{5}(g, g, g, g, g)=-f_{1}(g) \cdot f_{4}(g, g, g, g)-f_{4}(g, g, g, g) \cdot f_{1}(g)+d f_{5}(g, g, g, g, g)$.

This is not a null-homotopic map and we obtain

$$
m_{5}(g, g, g, g, g)=\mathbf{1}
$$

with $f_{5}(g, g, g, g, g)=0$.

Finally we consider the inductive computation of $m_{k}(g, g, \ldots)$ for $k>5$. It is easy to show that $f_{k}(g, g, \ldots, \mathbf{1}, \ldots, g, g)=0$. Therefore the only contribution to the left side of $(3.3)$ is $i m_{n}$. All terms of the form $f_{k}$. $f_{l}$ will vanish for $k+l>5$ on the right-hand side. It follows that $m_{k}(g, g, \ldots)=0$ for all $k>5$.

\footnotetext{
${ }^{5} f_{2}$ is an odd degree operator so $\left(f_{1} \otimes f_{2}\right)(g \otimes g)=-f_{1}(g) \otimes f_{2}(g)$.
} 
We have found a very simple result. The only nonvanishing product in the $A_{\infty}$-algebra is (3.16). We may now apply the methods discussed in [8] to compute the superpotential. Because (3.16) is the only nonvanishing product we will have a superpotential that is purely sextic. Let us use $X$ and $Y$ to denote the two chiral superfields associated with the two Ext $^{1}$ 's in (3.11). One obtains the exact result

$$
W_{4 \mathrm{~d}}=\operatorname{Tr}\left(\sum_{\sigma \in \mathfrak{S}_{6}} X_{\sigma(1)} X_{\sigma(2)} X_{\sigma(3)} Y_{\sigma(4)} Y_{\sigma(5)} Y_{\sigma(6)}\right)
$$

where $\mathfrak{S}_{6}$ is the symmetric group on six elements. This is consistent with the moduli space analyzed in [35] and the form conjectured ${ }^{6}$ in [34].

We illustrated this method of computing the superpotential for a very easy example that only required relatively small $8 \times 8$ matrices. Macaulay can cope with much more complicated cases where other methods, such as those in [34], would be impractical.

\subsection{The closed to open string map}

As a byproduct of the above analysis we find a very practical way of computing the closed to open string maps of $[36,37]$.

It was shown in [10] that, as far as correlation functions are concerned, there is no difference between a closed string associated to the monomial $m$ and an open string operator of the form $m . \mathbf{1}$. This map $m \mapsto m .1$ therefore yields the closed to open string map. This idea, and its consequences for the superpotential has also been discussed in [14].

We can immediately read this map from the Macaulay computations of $\operatorname{Ext}(M, M)$. So long as $M$ is not isomorphic to zero, the identity map will be an element of $\operatorname{Ext}^{0}(M, M)$ and thus is one of the generators given by Macaulay. The relations on this generator tell us exactly which monomials map to zero and each other under the closed to open string map.

For example, in the example above, 1 is annihilated by $I$ given by (3.10). So the closed strings given by monomials $x_{0}^{3} x_{2}^{2}$ and $x_{1}^{3} x_{2}^{2}$ would both map to the same nontrivial element of $\operatorname{Ext}^{2}(M, M)$ while $x_{0} x_{1} x_{2} x_{3} x_{4}$ maps to zero.

\footnotetext{
${ }^{6}$ We seem to have had a little more luck than the authors of [34] even though our computations are very similar. They did not obtain an exact sextic and needed to conjecture an $A_{\infty}$-isomorphism that would restore it to this form.
} 


\section{The linear $\sigma$-model interpretation}

\subsection{A conjecture}

The quotient construction of Section 2.3 has a very natural interpretation in terms of the gauged linear $\sigma$-model of [38]. This will lead us to the idea that different phases of the linear $\sigma$-model are associated to different, but equivalent, presentations of the category of topological B-type D-branes. The idea of using the gauged linear $\sigma$-model to analyze the phase structure of D-branes and the resulting categorical equivalences has also been explored recently in [39].

Let us consider a linear $\sigma$-model with a collection of chiral fields $\phi_{1}, \phi_{2}$, $\phi_{3}, \ldots \phi_{N}$. Let the gauge group be $\mathrm{U}(1)^{m}$. We also have a global U(1) Rsymmetry under which the superpotential, $\mathcal{W}$, has charge -2 . Thus, each chiral field has a collection of $m+1$ charges. Set

$$
S=k\left[\phi_{1}, \phi_{2}, \ldots, \phi_{N}\right]
$$

which will be a multi-graded algebra with $m+1$ gradings.

The equations of motion of the associated Lagrangian are divided into equations associated to the D-term and equations associated to the F-term. The D-terms impose a set of conditions that certain combinations of fields are not allowed to vanish. These conditions can be extracted from the triangulation of the fan associated to the phase of the linear $\sigma$-model as discussed in $[40]$.

The subset of $\mathbb{C}^{N}=\operatorname{Spec} S$ which is disallowed by the D-term equations of motion may be expressed in terms of an ideal $J \subset S$. This is the same ideal (which was denoted $B$ by Cox) that appears in [41].

The category $\mathbf{D}(\mathrm{gr}-S)$ is triangulated and, therefore, has a shift functor [...]. In addition, there are $m+1$ twist functors that cause a shift in the multigrading. These are normally denoted by parentheses (...).

The category $\operatorname{DGr} S(\mathcal{W})$ is the homotopy category of matrix factorizations of $\mathcal{W}$ over the ring $S$. Following [18], one may show that

$$
\operatorname{DGr} S(\mathcal{W}) \cong \frac{\mathbf{D}\left(\operatorname{gr}-S^{\prime}\right)}{\mathfrak{P e r f}\left(S^{\prime}\right)},
$$

where $S^{\prime}=S /(\mathcal{W})$.

The category $\operatorname{DGr} S(\mathcal{W})$ has the homological degree identified with one of the gradings. This latter grading is the one we identified with the R-charge. So $\operatorname{DGr} S(\mathcal{W})$ has one shift functor [...] and $m$ twist functors. 
We introduce the following notation. Let $T$ be an object in $\mathbf{D}($ gr- $S)$ which is annihilated by $\mathcal{W}$. Then $T$ is an object in $\mathbf{D}\left(\mathrm{gr}-S^{\prime}\right)$ and thus, by (4.2), an object in $\operatorname{DGr} S(\mathcal{W})$. Consider the collection of all shifted and twisted objects $T[\ldots](\ldots)$ and then find the minimal full triangulated subcategory of $\operatorname{DGr} S(\mathcal{W})$ that contains this collection. We denote the resulting subcategory $T^{\triangle}$. In other words, $T^{\triangle}$ is formed by iteratively applying the mapping cone construction to collections of $T$ and all of its translates and twists.

We now claim the following:

Conjecture 4.1. In any given phase of the gauged linear $\sigma$-model, the category of topological B-type D-branes is given by the triangulated quotient

$$
\frac{\operatorname{DGr} S(\mathcal{W})}{T^{\triangle}}
$$

where $T=S /(J+(\mathcal{W}))$.

First we show that this conjecture is true for the quintic. Here $S=$ $\left[p, x_{0}, \ldots, x_{4}\right]$ and the superpotential may be chosen as

$$
\mathcal{W}=p W=p\left(x_{0}^{5}+x_{1}^{5}+x_{2}^{5}+x_{3}^{5}+x_{4}^{5}\right) .
$$

We may choose the charges of our superfields to be

\begin{tabular}{c|cccccc} 
& $p$ & $x_{0}$ & $x_{1}$ & $x_{2}$ & $x_{3}$ & $x_{4}$ \\
\hline$R$ & -2 & 0 & 0 & 0 & 0 & 0 \\
$\mathrm{U}(1)$ & -5 & 1 & 1 & 1 & 1 & 1
\end{tabular}

This should look strikingly familiar to Section 2.2. This is the bigrading structure given to the ring $S$ for the quintic where $p$ plays the rôle of $X_{1}$.

As in earlier sections, we set $A=k\left[x_{0}, \ldots, x_{4}\right] /(W)$. We now claim that $\operatorname{DGr} S(\mathcal{W})$ is equivalent to the category $\mathbf{D}(\operatorname{gr}-A)$. A functor $\mathbf{D}(\operatorname{gr}-A) \rightarrow$ $\operatorname{DGr} S(\mathcal{W})$ is essentially constructed in Section 2.2. The inverse functor $\operatorname{DGr} S(\mathcal{W}) \rightarrow \mathbf{D}(\operatorname{gr}-A)$ can be constructed by taking a matrix factorization and setting $p=0$ to obtain $d_{o}=d_{C}$, which yields a free $B$-module resolution of an object in $\mathbf{D}(\mathrm{gr}-A)$.

The D-term equations depend on a parameter corresponding to the complexified Kähler form. If $X$ is "small", that is we are in the Landau-Ginzburg phase, then $p=0$ is excluded. That is, $J=J+(\mathcal{W})$ is the principal ideal $(p)$. The triangulated quotient is particularly simple when $T$ is the quotient of $S$ by a principal ideal as seen as follows. $S /(p)$ itself can be viewed as 
the cokernel of a morphism which looks like multiplication by $p$. Similarly all translates and shifts in grading of $S /(p)$ can be written as a cokernel of multiplication by $p$. It follows that $T^{\triangle}$ in this case consists of mapping cones of maps that involve multiplication by $p$. These maps are localized simply by setting $p$ equal to a unit.

Hence we perform the quotient in the conjecture simply by setting $p$ equal to some unit, say -1 . It is then clear that $\operatorname{DGr} S(\mathcal{W})$ becomes $\operatorname{DGr} B(W)$ under this quotient. So the conjecture is correct in the LandauGinzburg phase.

In the other "large radius limit" phase, the D-terms demand that $x_{0}, \ldots, x_{4}$ cannot simultaneously vanish, i.e., $J=\left(x_{0}, \ldots, x_{4}\right)$. Now, forgetting about the $p$-action, $(S / J)$ is equal to tors- $A$, as discussed in [19], for example. Here tors- $A$ denotes "torsion" modules as defined in [18]. It is then a result of Serre [42] that the bounded derived category of coherent sheaves on $X$ is the quotient $\mathbf{D}(\operatorname{gr}-A) /$ tors- $A$ (see also [18]). Thus the conjecture yields the desired result in both phases for the quintic threefold.

This conjecture can also be shown to be true for some of the phases associated to hypersurfaces in weighted projective spaces. This is most easily demonstrated by an example. Consider the linear $\sigma$-model with

$$
\mathcal{W}=p\left(x_{0}^{4}+x_{1}^{4}+x_{2}^{4}+t^{4} x_{3}^{8}+t^{4} x_{4}^{8}\right),
$$

and charges

\begin{tabular}{c|ccccccc} 
& $p$ & $t$ & $x_{0}$ & $x_{1}$ & $x_{2}$ & $x_{3}$ & $x_{4}$ \\
\hline$R$ & -2 & 0 & 0 & 0 & 0 & 0 & 0 \\
$Q_{1}$ & -8 & 0 & 2 & 2 & 2 & 1 & 1 \\
$Q_{2}$ & 0 & -2 & 0 & 0 & 0 & 1 & 1
\end{tabular}

In the Landau-Ginzburg phase $J=(p)(t)$ and the quotient in the conjecture sets $p$ and $t$ to units. Setting $t$ equal to a constant removes the $Q_{2}$-grading. Setting $p$ equal to -1 performs the quotient in Section 2.3. Thus we end up with the category of matrix factorizations of

$$
W=x_{0}^{4}+x_{1}^{4}+x_{2}^{4}+x_{3}^{8}+x_{4}^{8},
$$

as expected.

In the "orbifold" phase $J=(t)\left(x_{0}, \ldots, x_{4}\right)$. We again set $t$ equal to a constant removing the $Q_{2}$-grading. This part of the quotient leaves us with $\mathbf{D}(\operatorname{gr}-A)$ where $A=k\left[x_{0}, \ldots, x_{4}\right] /(W)$. Then we divide out by the category of torsion modules. What we obtain is the bounded derived category 
of coherent sheaves on the hypersurface $W=0$ in the weighted projective stack $\mathbb{P}_{\{2,2,2,1,1\}}^{4}$ as discussed in Proposition 2.16 of [18]. This is the expected result.

In the large radius phase the D-terms impose the constraints that $\left(x_{3}, x_{4}\right)$ $\neq(0,0)$ and $\left(x_{0}, x_{1}, x_{2}, t\right) \neq(0,0,0,0)$. The category of modules we are left with is still doubly graded using both $Q_{1}$ and $Q_{2}$. This exactly reproduces Cox's description of the derived category of coherent sheaves, as described in [41], on the hypersurface in the toric variety corresponding to the blow-up of the above orbifold. Thus we again obtain the expected result.

The above three paragraphs easily generalize to the case of any weighted projective space. The Landau-Ginzburg, large radius and (partially resolved) orbifold phases are all in compliance with Conjecture 4.1.

What is new would be the so-called hybrid cases. In the case of (4.8) we have a hybrid $\mathbb{P}^{1}$ phase where the conditions imposed are that $p=-1$ and that $\left(x_{3}, x_{4}\right) \neq(0,0)$. Imposing $p=-1$ will impose a quotient like in Section 2.3 and therefore gives us some kind of matrix factorization picture. Imposing $\left(x_{3}, x_{4}\right) \neq(0,0)$ is similar to quotienting by torsion modules and therefore has some of the character of coherent sheaves. Therefore, as expected we have some kind of hybrid picture of geometry and LandauGinzburg theories. We will not investigate this further here but it would be interesting to work out further details.

\subsection{Complete intersections}

As another application of Conjecture 4.1 we may consider cases of complete intersections in toric varieties that have a Landau-Ginzburg phase. Consider, for example, the linear $\sigma$-model with a superpotential

$$
\mathcal{W}=p\left(x_{0}^{3}+x_{1}^{3}+x_{2}^{3}+x_{3}^{3}\right)+q\left(x_{0} y_{0}^{3}+x_{1} y_{1}^{3}+x_{2} y_{2}^{3}\right)
$$

and charges

\begin{tabular}{c|ccccccccc} 
& $p$ & $q$ & $x_{0}$ & $x_{1}$ & $x_{2}$ & $x_{3}$ & $y_{0}$ & $y_{1}$ & $y_{2}$ \\
\hline$R$ & -2 & -2 & 0 & 0 & 0 & 0 & 0 & 0 & 0 \\
$Q_{1}$ & -3 & -1 & 1 & 1 & 1 & 1 & 0 & 0 & 0 \\
$Q_{2}$ & 0 & -3 & 0 & 0 & 0 & 0 & 1 & 1 & 1
\end{tabular}

$S$ is the ring $k\left[p, q, x_{0}, x_{1}, x_{2}, x_{3}, y_{0}, y_{1}, y_{2}\right]$ with a tri-graded structure given by these charges. 
In the large radius phase we note that Section 2.2 implies that $\operatorname{DGr} S(\mathcal{W})$ is equivalent to $\mathbf{D}(\mathrm{gr}-A)$ where

$$
A=\frac{k\left[x_{0}, x_{1}, x_{2}, x_{3}, y_{0}, y_{1}, y_{2}\right]}{\left(W_{p}, W_{q}\right)}
$$

and

$$
\begin{aligned}
& W_{p}=x_{0}^{3}+x_{1}^{3}+x_{2}^{3}+x_{3}^{3}, \\
& W_{q}=x_{0} y_{0}^{3}+x_{1} y_{1}^{3}+x_{2} y_{2}^{3}
\end{aligned}
$$

The D-terms imply that $J$ is the ideal $\left(x_{0}, x_{1}, x_{2}, x_{3}\right) \cdot\left(y_{0}, y_{1}, y_{2}\right)$. By Conjecture 4.1 the desired D-brane category is then

$$
\frac{\mathbf{D}(\operatorname{gr}-A)}{(A / J)^{\triangle}}
$$

By Cox's construction this is precisely the bounded derived category of coherent sheaves on $X$ where $X$ is Schimmrigk's manifold [43] defined by $W_{p}=W_{q}=0$ in $\mathbb{P}^{3} \times \mathbb{P}^{2}$.

In the Landau-Ginzburg phase we set $p=q=-1$. This collapses the tri-graded structure of $S$ to a single grading. Thus, if $M$ is an element of $\mathbf{D}(\mathrm{gr}-A)$ we now have the relation

$$
M[2]=M(3,0)=M(1,3) .
$$

It is not immediately obvious how to generalize Orlov's constructions $[18,28]$ to the multiply graded case and we will not try to make any statements about $\mathbf{D}_{\mathrm{Sg}}^{\mathrm{gr}}(A)$. Instead we note that we can perform the necessary quotient by using the method of Section 2.3 and therefore we know how to compute the necessary Ext groups by using Macaulay. ${ }^{7}$ It is also clear from (2.17) that, by setting $p=q=-1$ (that is, $X_{1}=X_{2}=-1$ ) we are forming matrix factorizations of $W_{p}+W_{q}$. Thus, the category of D-branes in this LandauGinzburg phase is again given by matrix factorizations.

We should note that not any complete intersection can be analyzed using matrix factorizations. This method is very much tied to the LandauGinzburg picture and this phase may not exist in general. For example, the

\footnotetext{
${ }^{7}$ As of Macaulay 2 version 0.9 .95 the necessary code for Ext is not written in a way that can handle multiple gradings. It is easy to rewrite the code in [21] such that it does. Future versions of Macaulay 2 are expected to be able to handle multiple gradings.
} 
intersection of two cubics in $\mathbb{P}^{5}$ has only a large radius Calabi-Yau phase and a hybrid $\mathbb{P}^{1}$ phase.

\section{Discussion}

In this paper we have shown that the matrix factorization approach to D-branes can be made quite practical for computations. Perhaps more interestingly, we have also seen how the phase picture of the linear $\sigma$-model naturally ties in with the derived category picture for D-branes. There are a number of directions for further research that present themselves.

In order to prove Conjecture 4.1 we would need to carefully analyze the boundary degrees of freedom in the linear $\sigma$-model and show that one requires a matrix factorization of $\mathcal{W}$. Then the conjecture should follow from imposing D-term conditions. Note, in particular, that one does not need to prove the appearance of the derived category directly by considering chain complexes of boundary conditions. There are analyses of linear $\sigma$-models with boundaries in the literature at present, such as [44,45], but these do not deal with matrix factorizations. More interestingly, the recent work of $[39,46]$ does perform an analysis along these lines and so promises a proof that we have a whole host of triangulated categories that are equivalent to each other, generalizing the McKay correspondence [47] and Orlov's equivalence [18].

Phases such as the hybrid models where one has a mixture of matrix factorizations and coherent sheaves might well be worthy of study in their own right.

Another interesting idea associated to the different phases is that of D-brane $\Pi$-stability. We know that $\Pi$-stability reduces to $\mu$-stability (modulo some subtleties [48]) for the large radius limit and that $\Pi$-stability reduces to $\theta$-stability for orbifolds. П-stability in the Landau-Ginzburg phase is not as well understood but some results were discussed in [23]. One might expect some specific flavor of stability that can be associated with each phase. This would be stated in terms of an abelian category that perhaps would be the heart of a $t$-structure of the triangulated category of D-branes in each case.

Clearly there are still many interesting properties of D-branes even in the case of topological field theory that have yet to be fully understood.

\section{Acknowledgments}

I wish to thank S. Katz, R. Plesser and A. Roy for useful discussions. The author is supported by an NSF grant DMS-0606578. 


\section{References}

[1] C. Vafa and N. Warner, Catastrophes and the classification of conformal theories, Phys. Lett. 218B (1989), 51-58.

[2] M. Kontsevich, Homological algebra of mirror symmetry, in Proceedings of the International Congress of Mathematicians, Birkhäuser, 1995, (120-139), alg-geom/9411018.

[3] E. Sharpe, D-branes, derived categories, and Grothendieck groups, Nucl. Phys. B561 (1999), 433-450, hep-th/9902116.

[4] M. R. Douglas, D-Branes, categories and $N=1$ supersymmetry, J. Math. Phys. 42 (2001), 2818-2843, hep-th/0011017.

[5] P. S. Aspinwall and A. E. Lawrence, Derived categories and zero-brane stability, JHEP 08 (2001), 004, hep-th/0104147.

[6] E. Witten, Chern-Simons gauge theory as a string theory, in 'The Floer Memorial Volume', eds. H. Hofer et al., Birkhäuser, Basel, 1995, 637-678, hep-th/9207094.

[7] M. Herbst, C. Lazaroiu and W. Lerche, Superpotentials, A-infinity relations and $W D V V$ equations for open topological strings, hepth/0402110.

[8] P. S. Aspinwall and S. Katz, Computation of superpotentials for DBranes, Commun. Math. Phys. 264 (2006), 227-253, hep-th/0412209.

[9] A. Kapustin and Y. Li, D-branes in Landau-Ginzburg models and algebraic geometry, JHEP 12 (2003), 005, hep-th/0210296.

[10] A. Kapustin and Y. Li, Topological correlators in Landau-Ginzburg models with boundaries, Adv. Theor. Math. Phys. 7 (2004), 727-749, hep-th/0305136.

[11] S. K. Ashok, E. Dell'Aquila and D.-E. Diaconescu, Fractional branes in Landau-Ginzburg orbifolds, Adv. Theor. Math. Phys. 8 (2004), 461-513, hep-th/0401135.

[12] I. Brunner, M. Herbst, W. Lerche and J. Walcher, Matrix factorizations and mirror symmetry: the cubic curve, hep-th/0408243.

[13] M. R. Douglas, S. Govindarajan, T. Jayaraman and A. Tomasiello, Dbranes on Calabi-Yau manifolds and superpotentials, Commun. Math. Phys. 248 (2004), 85-118, hep-th/0203173. 
[14] K. Hori and J. Walcher, F-term equations near gepner points, JHEP 01 (2005), 008, hep-th/0404196.

[15] K. Hori and J. Walcher, D-branes from matrix factorizations, Comptes Rendus Physique 5 (2004), 1061-1070, hep-th/0409204.

[16] I. Brunner and M. R. Gaberdiel, Matrix factorisations and permutation branes, JHEP 07 (2005), 012, hep-th/0503207.

[17] H. Enger, A. Recknagel and D. Roggenkamp, Permutation Branes and Linear Matrix Factorisations, JHEP 01 (2006), 087, hep-th/0508053.

[18] D. Orlov, Derived categories of coherent sheaves and triangulated catgeories of singularities, math.AG/0503632.

[19] P. Aspinwall, The Landau-Ginzburg to Calabi-Yau dictionary for D-branes, hep-th/0610209.

[20] D. R. Grayson and M. E. Stillman, Macaulay 2, a software system for research in algebraic geometry, available at http://www.math.uiuc.edu/Macaulay2/.

[21] L. L. Avramov and D. R. Grayson, Resulutions and cohomology over complete intersections, in 'Computations in Algebraic Geometry with Macaulay 2', eds. D. Eisenbud et al., Algorithms and Computations in Mathematics 8, Springer-Verlag, Berlin, 2001, 131-178.

[22] P. S. Aspinwall, D-Branes on Calabi-Yau manifolds, in 'Progress in String Theory. TASI 2003 Lecture Notes', ed. J. M. Maldacena, World Scientific, singapore, 2005, 1-152, hep-th/0403166.

[23] J. Walcher, Stability of Landau-Ginzburg branes, J. Math. Phys. 46 (2005), 082305, hep-th/0412274.

[24] D. Eisenbud, Homological algebra on a complete intersection, with an application to group representations, Trans. Amer. Math. Soc. 260 (1980), 35-64.

[25] J. Shamash, The Poincaré Series of a Local Ring, J. Algebra 12 (1969), 453-470.

[26] D. Eisenbud, Commutative algebra with a view towards algebraic geometry, Graduate Texts in Mathematics, 150, Springer, Berlin, 2004.

[27] S. I. Gelfand and Y. I. Manin, Homological algebra, Encyclopædia of Mathematical Sciences, 38, Springer, Berlin, 1994. 
[28] D. Orlov, Triangulated categories of singularities and D-branes in Landau-Ginzburg orbifolds, Proc. Steklov Inst. Math. 246 (2004), 227-248, math.AG/0302304.

[29] M. Kontsevich and Y. Soibelman, Homological mirror symmetry and torus fibrations, in 'Symplectic Geometry and Mirror Symmetry', eds. K. Fukaya et al., World Scientific, singapore, 2001, 203-263, math.SG/0011041.

[30] C. I. Lazaroiu, String field theory and brane superpotentials, JHEP 10 (2001), 018, hep-th/0107162.

[31] A. Tomasiello, A-infinity structure and superpotentials, JHEP 09 (2001), 030, hep-th/0107195.

[32] B. Keller, Introduction to A-infinity algebras and modules, Homology Homotopy Appl. 3 (2001), 1-35, math.RA/9910179.

[33] T. V. Kadeishvili, The Algebraic Structure in the Homology of an $A_{\infty}$ Algebra, Soobshch. Akad. Nauk. Gruzin. SSR 108 (1982), 249-252.

[34] S. K. Ashok, E. Dell'Aquila, D.-E. Diaconescu and B. Florea, Obstructed D-branes in Landau-Ginzburg orbifolds, hep-th/0404167.

[35] A. Albano and S. Katz, Lines of the Fermat quintic and the infinitesimal generalized Hodge conjecture, Trans. AMS 324 (1991), 353-368.

[36] C. Hofman and W.-K. Ma, Deformations of topological open strings, JHEP 01 (2001), 035, hep-th/0006120.

[37] C. I. Lazaroiu, On the structure of open-closed topological field theory in two dimensions, Nucl. Phys. B603 (2001), 497-530, hep-th/0010269.

[38] E. Witten, Phases of $N=2$ theories in two dimensions, Nucl. Phys. B403 (1993), 159-222, hep-th/9301042.

[39] M. Herbst, K. Hori and D. Page, various lectures and presentations, see www .msri.org/communications/vmath/VMathVideos/VideoInfo/ 2468 / show_video, www. theorie.physik. uni-muenchen.de/ ${ }^{\text {strings }}$ /pgst/index.php?target=talks, math.ias.edu/pages/activities / conferencesworkshops / workshops / workshop-on-homologicalmirror-symmetry-and-applications-i.php.

[40] P. S. Aspinwall, B. R. Greene and D. R. Morrison, Calabi-Yau moduli space, mirror manifolds and spacetime topology change in string theory, Nucl. Phys. B416 (1994), 414-480. 
[41] D. A. Cox, The homogeneous coordinate ring of a toric variety, J. Algebraic Geom. 4 (1995), 17-50, alg-geom/9210008.

[42] J. P. Serre, Modules projectifs et espace fibrés à fibre vectorielle, Séminaire Dubreil-Pisot 23 (1958), 531-543.

[43] R. Schimmrigk, A new construction of a three generation Calabi-Yau manifold, Phys. Lett. B193 (1987), 175-180.

[44] K. Hori, Linear models of supersymmetric D-branes, in 'Symplectic Geometry and Mirror Symmetry: Proceedings of the 4th KIAS Annual International Conference', eds. K. Fukaya et al., World Scientific, singapore, 2001, 111-186, hep-th/0012179.

[45] S. Hellerman and J. McGreevy, Linear sigma model toolshed for D-brane physics, JHEP 10 (2001), 002, hep-th/0104100.

[46] M. Herbst, K. Hori and D. Page, Phases of $N=2$ theories in $1+1$ dimensions with boundary, Toronto/Hamburg 2007 preprint, arXiv:0803.2045.

[47] T. Bridgeland, A. King and M. Reid, Mukai implies McKay, J. Amer. Math. Soc. 14 (2001), 535-554, math.AG/9908027.

[48] H. Enger and C. A. Lutken, Non-linear Yang-Mills instantons from strings are pi-stable D-branes, Nucl. Phys. B695 (2004), 73-83, hepth/0312254.

Center for Geometry and Theoretical Physics

Box 90318,

DUKE UNIVERSITY

Durham, NC 27708-0318, USA

E-mail address: psa@cgtp.duke.edu

Received August 16, 2007 